\title{
STRESS ANALYSIS OF HIGH SPEED FOUR BAR MECHANISM
}

\section{SREEKANTH SURA \& ALKA SAWALA}

Assistant Professor, Department of Aeronautical Engineering, MLR Institute of Technology, Hyderabad, India

The multi-body four bar mechanical system is an rigid links assemblage of assumed revolute joints and crank rotating motion for complete cycle, the links which produce relative motion. In higher speed mechanisms the assumption of rigid body links will not be valid, as the links are subjected to grater deformations. The high-speed activation of four bar mechanism demands that the members of the operating mechanism be tough, durable, flexible and safe to withstand axial stresses and undue vibrations. The analysis of the four bar mechanism can be performed by using kinematic coefficients method and theoretical vector loop, multi body dynamic analysis soft wares and by using Video graphic analysis etc,. Commonly such mechanisms are analyzed using CAD packages, e.g. ANSYS, ADAM etc. The stress analysis of a four bar mechanism (at higher operating speeds) is analyzed to find the limiting high speed of the four bar mechanism and the results are presented.
\end{abstract}

KEYWORDS: ADAMS Software, Four Bar Mechanism \& Multi Body Links Dynamics

Received: Nov 26, 2017; Accepted: Dec 15, 2017; Published: Jan 05, 2018; Paper Id.: IJMPERDFEB201823

\section{INTRODUCTION}

The four bar is the most useful mechanism which run at high speeds and should be considered as flexible to maintain accuracy, to operate in its speed regime and prevent the failure arising out of stresses due to dynamic forces involved. The speed of the mechanism at which the inertia forces arise cannot be neglected and therefore have to be considered.

The mechanism operates at predetermined speeds. The high speed activation and rapid cycle completion demands that the members of the operating mechanism to be safe, tough and durable to withstand stresses. The bending stress response of four bar links is analyzed using multi-body dynamics $[2,3]$.

\section{Scope of Work}

The bending stress response of a four bar mechanism is analyzed by simulating links throughout complete cycle, using Flexible multi-body dynamic analysis software ADAMS and the results are compared with results from earlier analysis of paper. After ascertaining the accuracy, the dynamic response of the four bar mechanism is analyzed for a scaled fabricated model.

\section{Four Bar Mechanism Analysis}

A four bar mechanism is considered to be a basic mechanism for preliminary analysis to evaluate the procedure to be applied for any complex mechanisms. 

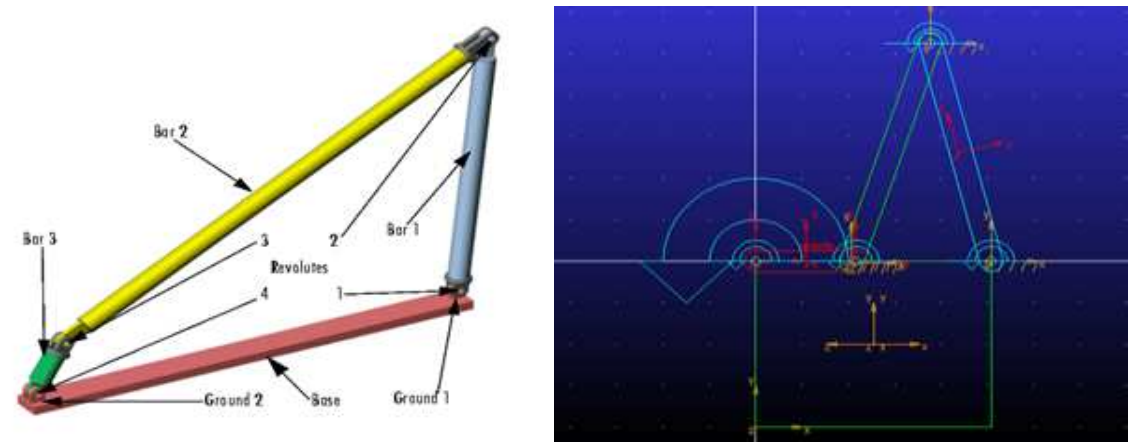

Figure 1: Four Bar Mechanism.

Figure 2: Four Bar Mechanism in ADAMS Software

The dimensions of the considered four bar mechanism is listed in below Table.

Table 1: Material and Geometric Properties of Four-Bar Mechanism

\begin{tabular}{|l|c|c|c|}
\hline \multicolumn{1}{|c|}{ Parameter } & Crank & Coupler & Follower \\
\hline Length $(\mathrm{cm})$ & 10.80 & 27.94 & 27.05 \\
\hline Area $(\mathrm{cm} 2)$ & 1.077 & 0.406 & 0.406 \\
\hline $\begin{array}{l}\text { Area moment of } \\
\text { inertia }(\mathrm{cm} 4)\end{array}$ & $1.66 * 10-2$ & $8.674 * 10-4$ & $8.674 * 10-4$ \\
\hline $\begin{array}{l}\text { Modulus of } \\
\text { Elasticity GPa }\end{array}$ & 70 & 70 & 70 \\
\hline
\end{tabular}

The crank is given an input speed of $340 \mathrm{rpm}$. The angular velocities of midpoint of coupler and follower links are plotted and compared with results from an earlier paper[2] as shown in figures.

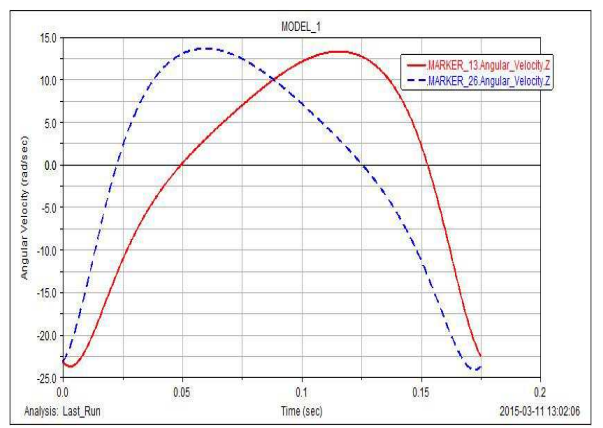

Figure 3: Angular Velocity Curves from Adams Software.

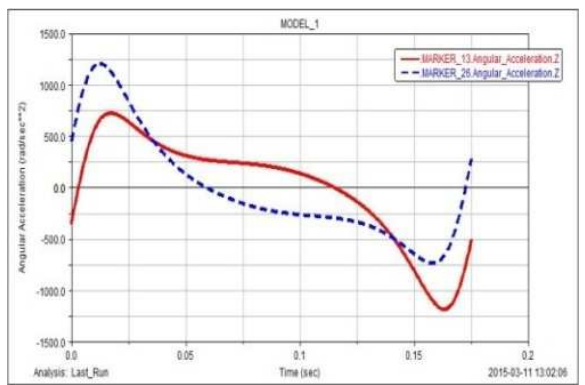

Figure 5: Angular Acceleration Curves from Adams Software

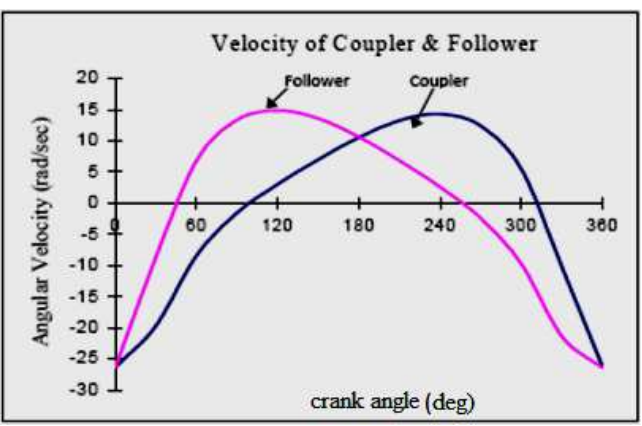

Figure 4: Theoretical Angular Velocity Curves (from Reference Paper\#2)

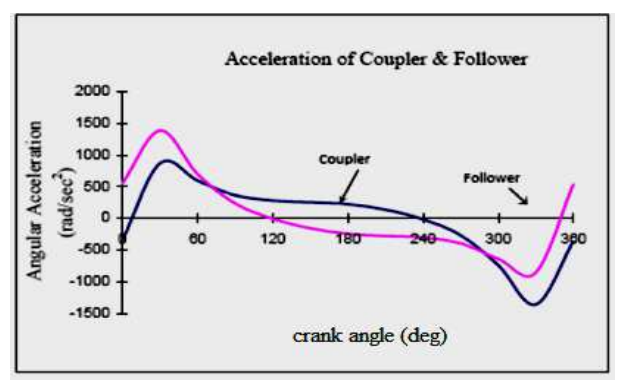

Figure 6: Theoretical Angular Acceleration Curves (from Reference Paper\#2) 


\section{BENDING STRESS ANALYSIS}

The maximum top bending stresses in mechanism copular (link-3) and follower (link-4) are listed and at particular node, the stress attains peck value and shows the maximum bending stress. The peck rise is shown in modal graphs. The two input motions are given as input at motion $1020 \mathrm{deg} / \mathrm{sec}$ and motions $2040 \mathrm{deg} / \mathrm{sec}$. the processor applied to complicated mechanism for better analysis purpose.

\begin{tabular}{|c|c|c|c|c|c|c|}
\hline \multicolumn{7}{|c|}{ VON MISES Hot Spots for PART_4_flex Date= 2015-04-11 16:11:26 } \\
\hline \multicolumn{2}{|c|}{ Model $=$.MODEL_ 1} & \multicolumn{2}{|c|}{ Analysis= Last_Run } & \multirow{2}{*}{\multicolumn{3}{|c|}{$\begin{array}{c}\text { Time }=0 \text { to } 0.356 \mathrm{sec} \\
\text { Radius }=0.0 \mathrm{~mm}\end{array}$}} \\
\hline \multicolumn{3}{|c|}{ Top 10 Hot Spots } & \multirow{2}{*}{$\begin{array}{l}\text { Abs } \\
\text { Time }\end{array}$} & & & \\
\hline Hot Spot & Stress & Node & & Location & wit LPRF ( & \\
\hline \# & (newton/mm*2) & id & (sec) & $\mathrm{x}$ & $\mathrm{Y}$ & z \\
\hline 1 & 29.1444 & 64 & 0.02848 & 219.071 & 130.634 & -2.5 \\
\hline 2 & 24.4325 & 51 & 0.02848 & 219.071 & 130.634 & 2.5 \\
\hline 3 & 24.0935 & 49 & 0.02848 & 209.783 & 168.137 & 2.5 \\
\hline 4 & 23.8502 & 67 & 0.02848 & 233.004 & 74.3793 & -2.5 \\
\hline 5 & 23.1642 & 24 & 0.02848 & 219.281 & 150.588 & 2.5 \\
\hline 6 & 22.5734 & 48 & 0.02848 & 205.139 & 186.889 & 2.5 \\
\hline 7 & 22.0134 & 62 & 0.02848 & 209.783 & 168.137 & -2.5 \\
\hline 8 & 21.5878 & 46 & 0.02848 & 195.851 & 224.392 & 2.5 \\
\hline 9 & 21.3824 & 53 & 0.02848 & 228.359 & 93.1309 & 2.5 \\
\hline 10 & 21.1099 & 63 & 0.02848 & 214.427 & 149.386 & -2.5 \\
\hline
\end{tabular}

Figure 7: Von Mises Stresses Table for Link-3 at Input Motion 1020deg/Sec

\begin{tabular}{|c|c|c|c|c|c|c|}
\hline \multicolumn{7}{|c|}{ VON MISES Hot Spots for PART_3_flex Date= 2015-04-11 16:11:26 } \\
\hline \multicolumn{2}{|c|}{ Model= .MODEL_1 } & \multicolumn{2}{|c|}{ Analysis $=$ Last_Run } & \multirow{2}{*}{\multicolumn{3}{|c|}{$\begin{array}{c}\text { Time }=0 \text { to } 0.356 \mathrm{sec} \\
\text { Radius }=0.0 \mathrm{~mm}\end{array}$}} \\
\hline \multicolumn{3}{|c|}{ Top 10 Hot Spots } & \multirow{2}{*}{$\begin{array}{l}\text { Abs } \\
\text { Time }\end{array}$} & & & \\
\hline Hot Spot & Stress & Node & & Location & wit LPRF & nm) \\
\hline \# & (newton/mm*2) & id & $(\mathrm{sec})$ & $\mathrm{x}$ & Y & $\mathrm{z}$ \\
\hline 1 & 22.2236 & 62 & 0.02848 & 129.181 & 95.448 & -2.5 \\
\hline 2 & 21.2276 & 49 & 0.02848 & 129.181 & 95.448 & 2.5 \\
\hline 3 & 20.6587 & 48 & 0.02848 & 135.493 & 114.385 & 2.5 \\
\hline 4 & 20.6329 & 63 & 0.02848 & 122.869 & 76.5113 & -2.5 \\
\hline 5 & 20.5879 & 52 & 0.02848 & 110.244 & 38.6377 & 2.5 \\
\hline 6 & 19.7859 & 64 & 0.02848 & 116.556 & 57.5744 & -2.5 \\
\hline 7 & 19.3087 & 51 & 0.02848 & 116.556 & 57.5744 & 2.5 \\
\hline 8 & 19.2942 & 65 & 0.02848 & 110.244 & 38.6377 & -2.5 \\
\hline 9 & 19.0054 & 50 & 0.02848 & 122.869 & 76.5113 & 2.5 \\
\hline 10 & 18.6806 & 53 & 0.02848 & 103.932 & 19.7009 & 2.5 \\
\hline
\end{tabular}

Figure 9: Von Mises Stresses Table for Link-4 at Input Motion 1020deg/Sec.

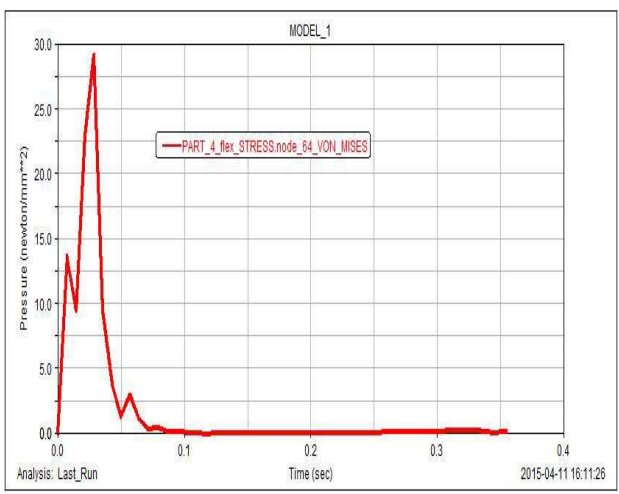

Figure 8: Von Mises Structural Bending Stress for Node-64,Link-3 At Input Motion 1020deg/Sec

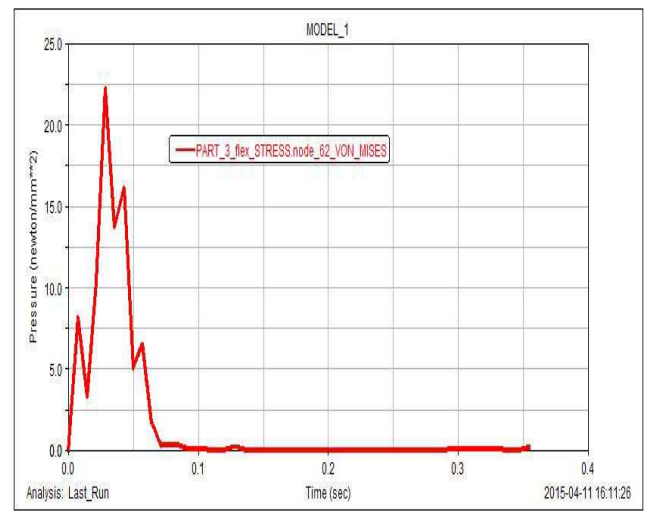

Figure 10: Von Mises Structural Bending Stress for Node-62,Link-4 at Input Motion 1020deg/Sec.

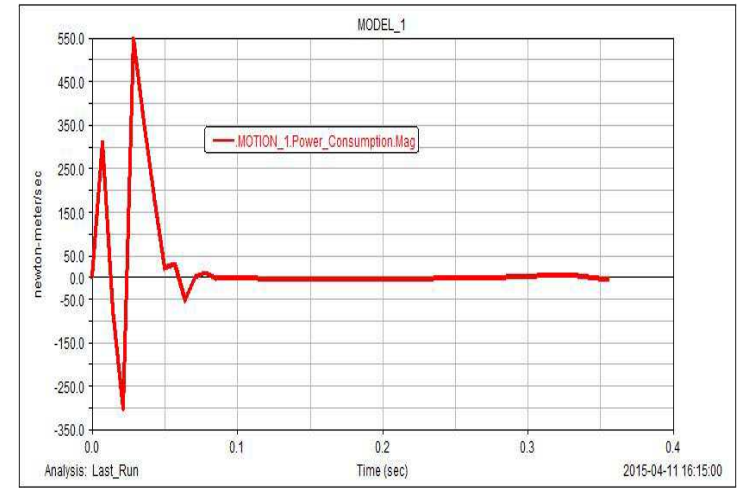

Figure 11: Required Input Power for Mechanism at 1020deg/Sec 


\begin{tabular}{|c|c|c|c|c|c|c|}
\hline \multicolumn{7}{|c|}{ VON MISES Hot Spots for PART_3_flex Date= 2015-04-11 16:02:59 } \\
\hline \multicolumn{2}{|c|}{ Model $=$.MODEL_1 } & \multicolumn{2}{|c|}{ Analysis= Last_Run } & \multirow{2}{*}{\multicolumn{3}{|c|}{$\begin{array}{c}\text { Time }=0 \text { to } 0.178 \mathrm{sec} \\
\text { Radius }=0.0 \mathrm{~mm}\end{array}$}} \\
\hline \multicolumn{3}{|c|}{ Top 10 Hot Spots } & \multirow{2}{*}{$\begin{array}{l}\text { Abs } \\
\text { Time }\end{array}$} & & & \\
\hline Hot Spot & Stress & Node & & Locatior & wrt LPRF ( & $\mathrm{mm})$ \\
\hline \# & $\left(\right.$ newton$\left./ \mathrm{mm}^{* * 2}\right)$ & id & $(\mathrm{sec})$ & $\mathrm{x}$ & $\mathrm{Y}$ & $z$ \\
\hline 1 & 35.0072 & 65 & 0.01424 & 110.244 & 38.6377 & -2.5 \\
\hline 2 & 33.9637 & 52 & 0.01424 & 110.244 & 38.6377 & 2.5 \\
\hline 3 & 32.893 & 64 & 0.01424 & 116.556 & 57.5744 & -2.5 \\
\hline 4 & 32.8713 & 66 & 0.01424 & 103.932 & 19.7009 & -2.5 \\
\hline 5 & 32.7298 & 27 & 0.01424 & 114.987 & 37.0565 & -2.5 \\
\hline 6 & 32.62 & 53 & 0.01424 & 103.932 & 19.7009 & 2.5 \\
\hline 7 & 32.5398 & 14 & 0.01424 & 114.987 & 37.0565 & 2.5 \\
\hline 8 & 32.1681 & 63 & 0.01424 & 122.869 & 76.5113 & -2.5 \\
\hline 9 & 32.1374 & 28 & 0.01424 & 121.3 & 55.9933 & -2.5 \\
\hline 10 & 32.0265 & 32 & 0.01424 & 146.549 & 131.74 & -2.5 \\
\hline
\end{tabular}

Figure 12: Von Mises Stresses Table for Link-3 at Input Motion 2040deg/Sec

\begin{tabular}{|c|c|c|c|c|c|c|}
\hline \multicolumn{7}{|c|}{ VON MISES Hot Spots for PART_4_flex Date= 2015-04-11 16:02:59 } \\
\hline \multicolumn{2}{|c|}{ Model= .MODEL_1 } & \multicolumn{2}{|c|}{ Analysis $=$ Last_Run } & \multirow{2}{*}{\multicolumn{3}{|c|}{$\begin{aligned} \text { Time } & =0 \text { to } 0.178 \mathrm{sec} \\
\text { Radius } & =0.0 \mathrm{~mm}\end{aligned}$}} \\
\hline \multicolumn{3}{|c|}{ Top 10 Hot Spots } & \multirow{2}{*}{$\begin{array}{l}\text { Abs } \\
\text { Time }\end{array}$} & & & \\
\hline Hot Spot & Stress & Node & & \begin{tabular}{|l} 
Location \\
\end{tabular} & wit LPRF & \\
\hline \# & (newton/mm*2) & id & (sec) & $x$ & Y & z \\
\hline 1 & 33.3581 & 46 & 0.01424 & 195.851 & 224.392 & 2.5 \\
\hline 2 & 31.686 & 64 & 0.00356 & 219.071 & 130.634 & -2.5 \\
\hline 3 & 31.0179 & 48 & 0.01424 & 205.139 & 186.889 & 2.5 \\
\hline 4 & 30.7875 & 49 & 0.01424 & 209.783 & 168.137 & 2.5 \\
\hline 5 & 30.3045 & 47 & 0.01424 & 200.495 & 205.641 & 2.5 \\
\hline 6 & 29.8821 & 61 & 0.01424 & 205.139 & 186.889 & -2.5 \\
\hline 7 & 29.3867 & 62 & 0.01424 & 209.783 & 168.137 & -2.5 \\
\hline 8 & 29.2315 & 60 & 0.01424 & 200.495 & 205.641 & -2.5 \\
\hline 9 & 29.1262 & 51 & 0.00356 & 219.071 & 130.634 & 2.5 \\
\hline 10 & 28.2374 & 63 & 0.01424 & 214.427 & 149.386 & -2.5 \\
\hline
\end{tabular}

Figure 14: Von Mises Stresses Table for Link-4 at Input Motion 2040deg/Sec

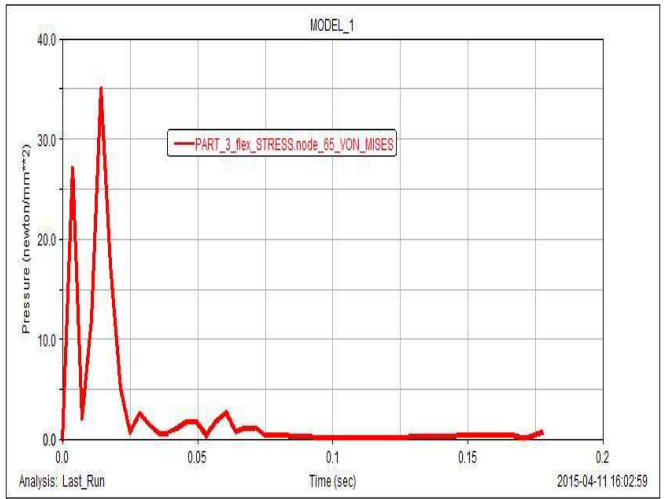

Figure 13: Von Mises Structural Bending Stress for Node65, Link-3 at Input Motion 2040deg/Sec

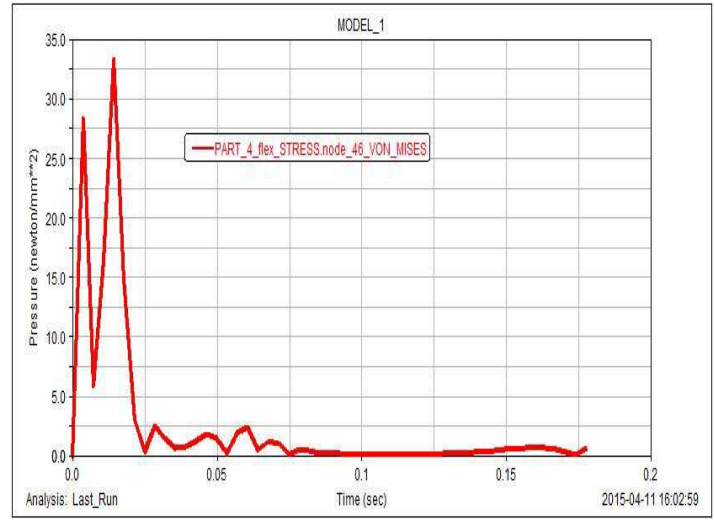

Figure 15: Von Mises Structural Bending Stress for Node-46 Link-4 at Input Motion 2040deg/Sec

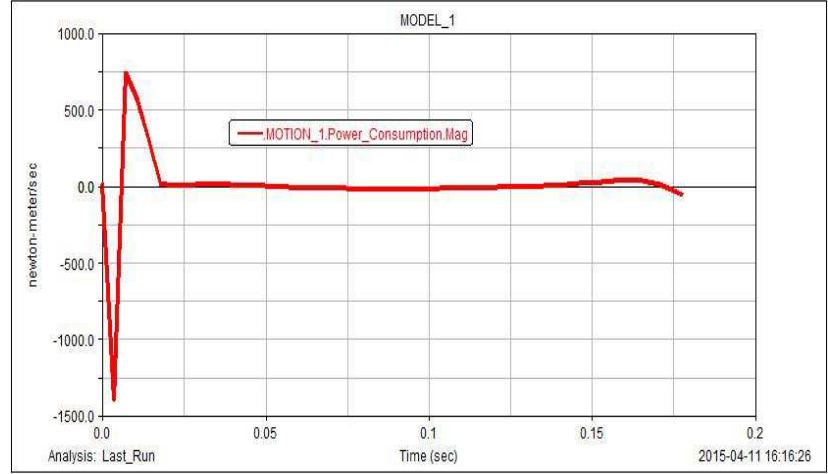

Figure 16: Required Input Power for Mechanism at 2040deg/Sec

\section{RESULTS AND DISCUSSIONS}

\section{Stress Analysis of Four-Bar Mechanism}

After assuring the kinematic parameters matched with the reference paper, it was further analyzed by using finite element method in ADAMS software, by making the links flexible. The input crank speeds applied is 1020 \& $2040 \mathrm{rpm}$. The stresses produced in the coupler and follower links are tabulated in Tables respectively. Also, the maximum stress variation at one node in coupler and follower links during one revolution of crank is shown. 
Table 2: Maximum Stresses on Coupler and Follower

\begin{tabular}{|l|c|c|c|c|c|c|}
\hline \multicolumn{1}{|c|}{ Links } & $\begin{array}{c}\text { Speed } \\
(\mathbf{r p m})\end{array}$ & $\begin{array}{c}\text { Node } \\
\text { Number }\end{array}$ & $\begin{array}{c}\text { Von Mises Stress } \\
\text { N/Mm2 }\end{array}$ & $\begin{array}{c}\text { Speed } \\
(\mathbf{r p m})\end{array}$ & $\begin{array}{c}\text { Node } \\
\text { Number }\end{array}$ & $\begin{array}{c}\text { Von Mises } \\
\text { Stress } \\
\text { N/Mm2 }\end{array}$ \\
\hline $\begin{array}{l}\text { Coupler } \\
\text { (link-3) }\end{array}$ & 1020 & 64 & 29 & 2040 & 65 & 35 \\
\hline $\begin{array}{l}\text { Follower } \\
\text { (link-4) }\end{array}$ & 1020 & 62 & 22 & 2040 & 46 & 33 \\
\hline
\end{tabular}

\section{CONCLUSIONS}

- In this work, proceeding with validation of analysis procedure for the dynamic analysis of the four bar mechanism is observed to be appropriate for using Multi body dynamic analysis software ADAMS in the analysis of high speed four-bar mechanism.

- The four-bar mechanism which is considered to be operated at high speeds is analyzed and after checking the induced stresses in all the links of the mechanism during its operation, maximum stress is found as $35 \mathrm{~N} / \mathrm{mm} 2$ when it operates at $2040 \mathrm{deg} / \mathrm{sec}$.

- It is concluded that if that mechanism operates beyond this high speed, the stresses induced may become more than the limiting values of the considered material, whose limiting value is about $35 \mathrm{~N} / \mathrm{mm} 2$, and hence it can be concluded that, the limiting speed of this particular mechanism is $2040 \mathrm{deg} / \mathrm{sec}$.

- Also this work procedure can be extended to similar mechanisms to decide the limiting high operating speed beyond which, when they are operated, produces the stresses beyond their limiting values. This work further can be extended by considering friction and damping effect on the analysis of mechanisms.

\section{REFERENCES}

1. Fu-Chen Chen, Yih-Fong Tzeng," On the dynamics of spring-type operating mechanism for $69 \mathrm{KV}$ SF6 gas insulated circuit breaker in open operation", Computers and Structures, 80 (2002) 1715-1723

2. Vaidya.M and Padole P.M," A Performance Evaluation of Four Bar Mechanism Considering Flexibility of Links and Joints Stiffness" The Open Mechanical Engineering Journal, 2010, 4, 16-24.

3. Amirouche FML. Computational methods in multi-body dynamics. Englewood Cliffs, New Jersey: Prentice-Hall, Inc; 1992.

4. Greenwood DT. Principles of dynamics. Englewood Cliffs, New Jersey: Prentice-Hall, Inc; 1988.

5. Anjaneyulu.J, VenkataRao.G, and Krishnamohan Rao.G "Theoretical And experimental Dynamic Analysis Of High Speed Mechanism Of A Switch Gear" International Journal of Applied Engineering Research, ISSN 0973-4562 Volume 10, Number 2 (2015) pp. 5271-5283, Research India Publications. http://www.ripubilcation.com.

6. Manish Mehta \& P M George, Elastodynamic Analysis of Four Bar Mechanism Using Matlab and Ansys WB, International Journal of Mechanical and Production Engineering Research and Development (IJMPERD), Volume 2, Issue 3, August September 2012, pp. 76-82

7. Uicker Jr.J.J, Pennock.G.R, Shigley.J.E, Theory of Machines and Mechanisms, third ed., Oxford University Press, New York, 2003.

8. Ben Jonker, "Linearization Of Dynamic Equations Of Flexible Mechanisms-A Finite Element Approach" international journal for numerical methods in engineering vol.3.1, 1375- 1392 (1991). 
